\title{
Medical Technologies; Could this be the "Glue" to Improve the Relationship between Health Professionals, Patients and Healthcare Systems? A Perspective
}

\author{
Delanerolle G1, Will Goodison ${ }^{2}$, Elliot $\mathrm{K}^{3}$, Goroszeniuk $\mathrm{T}^{4}$, Phiri $\mathrm{P}^{5 *}$ and \\ Shetty $\mathbf{A}^{2,3}$ \\ ${ }^{1}$ University of Oxford, UK \\ ${ }^{2}$ University College London NHS Foundation Trust, UK \\ ${ }^{3}$ Southern Health NHS Foundation Trust, Research \& Development Department, UK \\ ${ }^{4}$ University College London Hospitals, UK \\ ${ }^{5}$ University College London, UK
}

Commentary

Volume 6 Issue 1

Received Date: March 17, 2021

Published Date: March 31, 2021

DOI: $10.23880 /$ pprij-16000271

*Corresponding author: Dr. Peter Phiri, Southern Health NHS Foundation Trust, Research \& Development Department. Clinical Trials Facility, Moorgreen Hospital Southampton, SO30 3JB, UK, Email: peter.phiri@southernhealth.nhs.uk

\section{A New World}

The COVID-19 pandemic has changed the landscape of modern-day medicine. One of the many lasting legacies of the pandemic may be the transformative effect it has had on digitising health care systems. Countries where digital technology was already integrated and established in healthcare systems were able to respond more quickly and flatten their respective incidence rates of Covid-19 in comparison to those that had more traditional methods of working [1]. For example, data tracking and the use of artificial intelligence (AI) in particular have proven effective in tracking the spread of disease and predicting new pockets of infection [1]. Additionally, the use of telemedicine consultations have nations scrambled to upgrade digital infrastructure and implement new strategies to cope with the demands posed by the pandemic [2]. However, the longterm implications at first hand appear to be positive, in that most healthcare systems would achieve a much-needed infrastructural upgrade that both patients and clinicians would benefit from in many different ways.

These changes equally mean the professional relationship between physician and patient would be transforming into the digital era that would be revolutionising healthcare. A primary reason for close interactions between doctor and patient has been driven by the need to be able to assess the patient more comprehensively and have an open dialogue to determine the most appropriate diagnosis and treatment although this is too changing. This also removes the need for patients to needlessly travel long distances. Society has reached a point where clinicians and patients alike, in theory, now possess the technology and infrastructure to diagnose and monitor responses, track treatments and even have face to face discussions without the need for a physical consulting room or clinical space. Nevertheless, does this allow the relationship to continue, as it currently exists? This may appear to be a simple question although to obtain a response may be cumbersome for a variety of reasons and worth exploring further.

\section{The New Normal}

The changing dynamics of the doctor-patient relationship have led us from paternalistic models to more patient centric models [3]. Whilst both aspects have advantages and disadvantages, it is imperative to evaluate each scenario in its own right in some ways. Greater patient autonomy over their health is as important as balancing the expertise provided by a clinician. New and emerging technologies have granted greater access to equitable knowledge [4], improved medical data collection and tracking [5], and medical services in a way that is convenient compared to some of the traditional service access models. Emerging technologies are equally simpler to use, with almost $90 \%$ of the global population having access to a mobile phones, application availability 


\section{Psychology \& Psychological Research International Journal}

to support healthcare requirements has become more convenient.

An important concern among clinicians is that digital technologies may introduce barriers between doctors and patients. Whilst a physical examination has always been an important dynamic of the relationship, building trust, confidence and providing an all-important human interaction underpins disease management [6]. It is important to emphasis that tele-medicine does not mean 'remote medicine' for every clinical scenario is the way forward. Technology could liberate patients and clinicians if used effectively. It can allow physicians and patients to track disease progression and symptomatology changes in real time [7]. This could further assist in developing early diagnosis methods through data science [8]. Virtual surgery is another possibility resulting from advancements in medical technologies that allows clinicians in developed countries to help those in low and middle-income countries [9]. The use of medical software for developing 3D models needed to assist surgeons is another important aspect, especially for trauma surgery. This could be utilised to reach remote areas or even war-torn countries, ultimately managing at least part of the team in a safer environment.

Whilst medical technologies could drive greater welfare for the public, there could be concerns raised around accountability and medical negligence as well. To mitigate some of these concerns virtual consultations could be triaged with prioritisation of patients to have face to face reviews.

Another important factor to consider here is that of healthcare professional associated occupational burnout, this could be managed in a better way. Medical technologies could manage some of the clinical pressures associated with clinics by addressing better allocation of appointments and patients could equally attend these regardless of any other competing commitments they have, especially those who are in full time work, and are likely to miss appointment. This could assist with saving costs associated with missed appointments. However, comprehensive evidence would be required to appropriately investigate the effects of using medical technologies and management of burnout by way of research. For example, within the UK, where it is reported a third of all doctors burnout [10], faces an exponential increase of resource burden as well as burnout despite the National Health Service (NHS) being considered as the $4^{\text {th }}$ largest workforce in the world. Equally, the NHS has struggled to adopt technological innovations in the historically [11].

Another facet to this would be the effects of the pandemic on staff due to poorly thought through and dysfunctional technologies, hastily developed and rolled out, could actually serve to exacerbate physician burnout [12]. Some clinical areas have benefitted more than others, such as Radiology, General Surgery, Trauma and Oncology, where technological advancement by way of software and hardware, including robotics have supported the advance of optimising patient care as well as limiting time spent using conventional methods. These have considerably helped with managing workflow and lists to a point where healthcare systems are able to better manage their population demand $[13,14]$. Medical technologies could very much be a supportive tool to the physician, enabling them to better support their wellbeing thus, enabling the improvement of staff retention rates and the overall management of patient access pathways that are resourced appropriately. This could further aid with alleviating pressures around funding for both primary, secondary and tertiary care systems.

Whilst there are many advantages to medical technologies, immediate economic viability may not always be possible. To achieve economic benefit and long-term sustainability, implementation science-based research to best manage these across healthcare systems would be required. This partly because, global healthcare systems have varying business models, thus, to agree on unified health-economic models would be challenging. Furthermore, conduct of clinical trials to test these medical technologies as well as their economic benefit would be important. However, this may be perceived, as an onerous task by many, as conducting comprehensive clinical research would require money and time. In order to gain the most cost effective and optimal use of medical technologies, especially wearable devices and sensors, mobile diagnostic devices and clinical grade imaging [10] would vastly benefit from pursuing clinical research methods to better understand patient and healthcare benefit.

\section{The Journey So Far}

The current wave of medical technology start-ups as well as major tech giants such as IBM Watson, Apple, Amazon, Google and Microsoft investing in researchers to develop medical technologies demonstrate the fast-paced digitisation of modern medicine. These companies have made using algorithms and automation an art form for the elderly whilst remaining 'trendy' to the younger audiences, and are keen to combine their expertise with existing health care systems. Whilst financial incentives [15] rather than altruistic motives may be at the heart of this expansion, the coders of Silicon Valley are well placed to manage the digital revolution of healthcare, embedding digital medicine methods into routine health care services, with research and development particularly in artificial intelligence a top priority [12].

There is also evidence, from many parts of the world, 


\section{Psychology \& Psychological Research International Journal}

about the use of chat bots and apps as well as other software that enhance the therapeutic relationship can be economically beneficial and could be a self-sustainable tool to ease some of the over-stretched nature of services in healthcare. However, advancements in technology and large investments could have a negative impact on the cost of healthcare that in a privatised healthcare system may create tiers of access. The United States, for example, has had increasing technological integration into services with subsequent exponential rises in the cost of care [16].

\section{The Cumbersome Ethical Conundrum}

There are a number of pragmatic and ethical concerns of course to consider ranging from technical requirements to governance around maintaining confidentiality during digital consultations. Furthermore, there are concerns by some clinicians and patients alike, that remote relationships could be impersonal. One of the ways that existing technology could be used constructively would be to adapt these to clinical settings. Mental health services have demonstrated some success for example, where millions of smartphone apps, both clinically developed and otherwise, have provided users with support from anxiety and stress by way of mindfulness techniques, diet and mood tracking. However, the lack of quality control and assurance aspects of these non-medically developed AI tools could have a negative effect on patients and the public's wellbeing long term. This could raise further ethical implications. It is reasonable to expect that medical technologies used in healthcare settings should have to pass rigours checks and scrutiny that is applied to normal medical research.

\section{Conclusions}

Medical technologies demonstrate much promise, although careful consideration should be made in regards to the implementation of the most effective and evidence-based applications that would be fit for purpose across varying healthcare systems. Whilst digital healthcare continues to influence clinical care, society and the global population, challenges remain in regards to its' personalisation to patients and legal as well as ethical implication. One thing is clear; technology in medicine is here to stay. We must adapt to it and assimilate it into our practice. If we take ownership, we have a chance to broaden and evolve the complex relationship that exists between physician and patient. The pandemic has pushed us full force into the digital age and it is our responsibility to consider now, and as part of all future training and development, the impact technology can have on our practice and how best to use it for our patient's advantage.

\section{References}

1. Whitelaw S, Mamas MA, Topol E, Van Spall HGC (2020) Applications of digital technology in COVID-19 pandemic planning and response. Lancet Digit Health 2(8): e435-e440.

2. Keesara S, Jonas A, Schulman K (2020) Covid-19 and Health Care's Digital Revolution. N Engl J Med 382(23): e82.

3. Stewart M (2005) Reflections on the doctor-patient relationship: from evidence and experience. $\mathrm{Br} J$ Gen Pract 55(519): 793-801.

4. Percheski C, Hargittai E (2011) Health informationseeking in the digital age. J Am Coll Health. 59(5): 379386.

5. Paré G, Leaver C, Bourget C (2018) Diffusion of the Digital Health Self-Tracking Movement in Canada: Results of a National Survey. J Med Internet Res 20(5): e177.

6. Horton R (2019) Offline: Touch-the first language. Lancet 394(10206): 1310.

7. Pevnick JM, Birkeland K, Zimmer R, Elad Y, Kedan I (2018) Wearable technology for cardiology: An update and framework for the future. Trends Cardiovasc Med 28(2): 144-150.

8. The Lancet Oncology (2018) Digital oncology apps: revolution or evolution? Lancet Oncol 19(8): 999.

9. Higginbotham G (2021) Virtual Connections: Improving Global Neurosurgery through Immersive Technologies. Front Surg 8: 629963.

10. McKinley N, McCain RS, Convie L, Clarke M, Dempster M, et al. (2020) Resilience, burnout and coping mechanisms in UK doctors: a cross-sectional study. BMJ Open 10(1): e031765.

11. Asthana S, Jones R, Sheaff R (2019) Why does the NHS struggle to adopt eHealth innovations? A review of macro, meso and micro factors. BMC Health Serv Res 19(1): 984.

12. Yates SW (2020) Physician Stress and Burnout. Am J Med 133(2): 160-164.

13. National Academies of Sciences, Engineering, and Medicine; National Academy of Medicine; Committee on Systems Approaches to Improve Patient Care by Supporting Clinician Well-Being (Washington (DC): National Academies Press (US); 2019) Taking Action Against Clinician Burnout: A Systems Approach to 
Psychology \& Psychological Research International Journal

Professional Well-Being.

14. Steinhubl SR, Muse ED, Topol EJ (2015) The emerging field of mobile health. Sci Transl Med. 7(283): 283rv3.

15. Cohen AB, Mathews SC, Dorsey ER, Bates DW, Safavi K
(2020) Direct-to-consumer digital health. Lancet Digit Health 2(4): e163-e165.

16. He J, Baxter SL, Xu J, Xu J, Zhou X, etal. (2019) The practical implementation of artificial intelligence technologies in medicine. Nat Med 25(1): 30-36. 